\title{
CUANDO CHARLES DARWIN PUBLICÓ EL ORIGEN DE LAS ESPECIES (1859)
}

\author{
WHEN CHARLES DARWIN PUBLISHED ON THE ORIGIN OF SPECIES (1859)
}

Vicente Pérez ${ }^{1}$

Se ha dicho que yo hablo de la selección natural como de un poder activo o divinidad.

Charles Darwin

El Origen de las Especies (Capítulo IV).

Aunque el Origen de las Especies no es el primer libro sobre evolución, en el sentido de que las especies pueden cambiar a través del tiempo, con sus seis ediciones revisadas (1859, 1860, 1861, 1866, 1869 y 1872, John Murray, Londres) y muchísimas reimpresiones, convirtió a Charles Darwin (1809-1882) en uno de los más influyentes pensadores de todos los tiempos y tuvo más de un efecto en la vida del ser humano que cualquiera otra obra no religiosa en la historia de la humanidad (Ghiselin \& Goldie 1997).

La idea de la evolución aparece en diversas obras, incluso de la antigüedad. Darwin utilizó la expresión descendencia con modificación, y que más tarde se conocería como evolución. El gran naturalista sólo empleó este último término en $E l$ Origen del Hombre (1871) y a partir de la quinta edición de El Origen de las Especies (1869).

Darwin no fue el inventor de la idea de la evolución, formulada, entre otros, por el naturalista francés Jean-Baptiste Lamarck (1744-1829) y por su propio abuelo Erasmus Darwin (1731-1802), pero supo, primero, organizar los argumentos para presentar a la evolución como un hecho, y, luego, proponer a la selección natural, como mecanismo de ella (Milner 1995), que él explicó por analogía con la crianza o cultivo selectivo en la agricultura, con materiales, razonamientos y ejemplos que había recopilado en su largo viaje alrededor del mundo a bordo del H.M.S. Beagle (1831-1836) bajo el mando del capitán Robert FitzRoy (1805-1865), y después de muchos años de reflexión.

Aunque casi sin discusión se atribuye la paternidad de la teoría de la selección natural a Darwin, más de una vez se han alzado voces que han propuesto que la teoría de la evolución por medio de la selección natural se denomine teoría de DarwinWallace (Milner 1995) o teoría de Wallace-Darwin siguiendo un procedimiento nomenclatural como en la ley de Hardy y Weinberg (Josa i Llorca 2008).

Las principales ideas que Darwin plasmaría en El Origen de las Especies las inició en un cuaderno de notas abierto en 1837; en 1842 ya tenía un bosquejo de 13 páginas, y en 1844 había hecho un resumen de 232 páginas, que fueron presentadas a la Linnean Society para su publicación en coautoría (1858) con Alfred Russel Wallace (1823-1913), que

1 Laboratorio de Entomología, Instituto de la Patagonia, Universidad de Magallanes. Casilla 113-D. Punta Arenas, Chile. vicente. perez@umag.cl 
había desarrollado la teoría de la selección natural trabajando independientemente. Aunque Darwin se refería siempre a "mi teoría", Wallace, con su modestia y caballerosidad habitual lo apoyaba ("es suya y sólo suya”) a partir de la quinta edición (1869).

Wallace llegó a concebir sus concepciones evolucionistas no desde la analogía con la selección artificial de animales domésticos, como Darwin, sino de sus observaciones en la distribución natural de vegetales y animales en el archipiélago de las Molucas (actualmente Malasia), cuyos resultados había comunicado a Darwin.

Temiendo que Wallace tomara la delantera, en trece meses, redactó, con 155.000 palabras lo que hoy conocemos como El Origen de las Especies, proyectado como un resumen de una obra más amplia que nunca concretó (Milner 1995).

\section{EL ORIGEN DE LAS ESPECIES}

El Origen de las Especies fue escrito para un público especializado: en su texto se citan 192 autores en los diferentes capítulos con sus descripciones de material biológico, descubrimientos, afirmaciones, opiniones; además, 34 autores en el análisis bibliográfico histórico (21 de ellos no citados en los capítulos) en el Bosquejo Histórico Sobre el Progreso de las Opiniones Sobre el Origen de las Especies Antes de la Publicación de la Primera Edición de esta Obra. En suma, Darwin se basó en una numerosa bibliografía. Y, para facilitar la lectura del texto, solicitó al entomólogo y traductor inglés William Sweetland Dallas (1824-1890) que confeccionara un Glosario que fue incorporado desde la primera edición de la obra.

Constituido en una de las grandes obras clásicas de la ciencia, era un libro denso y difícil en opinión de los mejores amigos de Darwin, tanto que éste, inseguro de que fuera bien recibido por los no especialistas, ofreció cubrir los gastos de su publicación. Sin embargo, la primera edición, de 1250 ejemplares se agotó en las librerías el primer día (24 de noviembre de 1859) y se ha reeditado continuadamente desde entonces (Milner 1995).

Actualmente no es un libro muy leído, excepto por biólogos profesionales. Sin embargo, es mucha la gente que emite opiniones sobre él, la gran mayoría de las veces injustificadas. Es conveniente traer a colación la frase de Huxley (1859a): "Aquellos que quieran juzgar el libro, deben leerlo".
El lenguaje empleado por Darwin en El Origen de las Especies es muy cuidadoso, para no herir susceptibilidades. En la introducción, manifiesta que lo observado por él en la distribución geográfica de los organismos en Sudamérica, asociada a su pasado y presente geológicos, parecían dar luz sobre el origen de las especies; que a las mismas conclusiones sobre el origen de las especies había llegado Wallace en el archipiélago malayo; que los naturalistas pueden llegar a la conclusión de que las especies no han sido independientemente creadas; que es errónea la idea de que cada especie ha sido creada independientemente; que su convencimiento es que las especies no son inmutables; e introduce la idea de que la selección natural ha sido el mecanismo más importante en la modificación de las especies.

A partir de esta presentación, desarrolla sus ideas manteniendo una respetuosa expresión sobre la teoría de la creación (creación independiente, acto especial de creación, creación ordinaria, con un total de 19 menciones), desarrolla el tema de la lucha por la existencia (lucha universal por la vida, rigurosa competencia, con 13 utilizaciones) y la supervivencia de los más aptos (3), para plantear su teoría de la descendencia con modificación (9, que más tarde se denominará evolución [término éste que utiliza 6 veces, pero solamente a partir de la quinta edición (1869), aunque usa evolucionado]), y que se logra mediante la selección natural (50 en total, en el resto del libro; además la trata especialmente: Capítulo IV. Selección natural o la supervivencia de los más aptos, y Capítulo VII. Objeciones diversas a la teoría de la selección natural).

Sus afirmaciones más fuertes serían las de que no es una explicación científica el aceptar que "fue una decisión del Creador construir todos los animales y plantas. En cada una de las grandes clases, según un plan uniforme"; y "que en el futuro...se proyectará mucha luz sobre el origen del hombre..."

\section{REACCIONES A LA PUBLICACIÓN DE EL ORIGEN DE LAS ESPECIES}

Se presentan las opiniones generadas por la publicación de la primera edición de El Origen, con párrafos escogidas de ellas, para mostrar cómo fue recibida la obra de Darwin por los científicos y público culto de la época victoriana y personalidades de la sociedad de la época en 1859 y 1860, de acuerdo a 
la bibliografía de Charles Darwin On-line (2009), por considerarse más útil que analizar las características del siglo que en que vivió el gran naturalista.

Se eligieron las revisiones publicadas en los dos años nombrados, porque por ser inmediatos a la aparición de El Origen, representan las opiniones más espontáneas de los autores. No se incluyeron Higgins (1860), porque realizó comentarios sobre comentarios, y tampoco Bronn (1860), porque el texto está en alemán.

Los datos biográficos de los autores corresponden a los registrados por Smith (1987), Milner (1995), Ghiselin \& Pope (1997) e Internet. Los que no se consignan es porque no han sido encontrados.

Quienes publicaron opiniones (1859-1860) sobre la recién aparecida obra de Darwin fueron beatos laicos y eclesiásticos, biólogos, botánicos, economistas, entomólogos, filósofos, fisiólogos, geólogos, paleontólogos y otros personajes cuyos antecedentes no se ha podido establecer, pero que comparten la capacidad de saber exponer sus ideas, guiándose por la reflexión o aferrándose a sus creencias religiosas:

- “...y cuando él presenta ante nosotros los resultados de 20 años de investigación y reflexión, debemos prestar atención aun cuando estemos dispuestos en contra" (Huxley 1859a).

Thomas Henry Huxley (1825-1895). Biólogo evolucionista inglés, especializado en Anatomía Comparada y Paleontología. Fue el primer reseñador de El Origen de las Especies (1859) en el London Times. Es recordado principalmente como un combativo defensor de Darwin (se le llamó "el dogo de Darwin").

- "La hipótesis del Sr. Darwin sobre el origen de las especies ocupará su lugar entre las teorías establecidas de la ciencia, tendrá sus consecuencias cualesquiera que sean. $\mathrm{Si}$, por otra parte, el $\mathrm{Sr}$. Darwin erró, sea de hecho o en razonamiento, sus seguidores pronto encontrarán los puntos débiles de su doctrina, y su extinción por alguna aproximación más cercana a la verdad ejemplificará su propio principio de la selección natural" (Huxley 1859b).

- "La falacia de la teoría del Sr. Darwin sobre el origen de las especies por medio de la selección natural, puede encontrarse en las primeras páginas de su libro, donde examina la diferencia entre los actos voluntarios y deliberados de selección aplicados metódicamente por el hombre en la crianza y desarrollo de las plantas cultivadas y las influencias casuales que pueden afectar animales y plantas en estado natural. El origen de toda la diversidad entre los seres vivos permanece como un misterio tan totalmente inexplicado, como si el libro del Sr. Darwin jamás hubiera sido escrito, porque ninguna teoría sin el soporte de los hechos, ...puede ser admitida por la ciencia" (Agassiz 1860).

Louis Agassiz (1807-1873). Nacido en Suiza, emigró a los Estados Unidos en 1846, ingresado como profesor a la Universidad de Harvard en 1847. Geólogo, zoólogo y anatomista comparativo, especialista en peces fósiles. Ridiculizó la teoría darwiniana en el momento de su aparición en 1859, y se negó a reconsiderar su postura hasta el fin de su vida.

- "El autor de este libro se esfuerza por establecer, aunque con una teoría diferente y un proceso de razonamiento algo diferente, la misma conclusión a la que llegaron el naturalista francés Lamarck y el autor inglés de los Vestigios de la Creación..., sin ninguna intervención de algún acto de poder creador" (Bowen 1860).

Francis Bowen (1811-1890). Filósofo y economista norteamericano, catedrático de la Universidad de Harvard. Dirigió desde 1843 a 1854 la North American Review, en la que publicó su apreciación sobre El Origen de las Especies.

- "En la mente filosófica del Sr. Darwin (hay pocos hombres de ciencia en nuestro país actualmente que pudieran merecer la honorable designación de filósofo) la idea primeramente fue cautelosamente recogida; gradualmente fue desarrollada de una forma sistemática y sometida a una diversidad de pruebas; y cuando su autor se satisfizo de su solidez, la aplicó por varios años durante el tiempo que su débil salud se lo permitió, a la labor de preparación de un trabajo que pudiera contener no sólo una exposición de sus puntos de vista, sino con una completa exposición de la evidencia en las cuales está basada" (Carpenter 1860a).

William Benjamín Carpenter (1813-1885). Fisiólogo y escritor científico inglés. Profesor en la Universidad de Londres. Autor de varios influyentes textos. 
- "Su atención [de Darwin] estuvo dirigida a la investigación de algunos hechos que le impresionaron en la distribución de los habitantes de Sudamérica, y en las relaciones geológicas de los habitantes del presente, y a las el pasado de ese continente durante el viaje a bordo del H.M.S. Beagle, del cual nos dio tan admirable Diario. Estos hechos le parecieron a él que arrojaban alguna luz sobre el origen de las especies - ese misterio de los misterios, como uno de nuestros más grandes filósofos lo denominado. Pero como es imposible, en la naturaleza de las cosas, obtener algún antecedente como evidencia positiva sobre los más remotos antecesores de la investigación, descartaremos para la presente toda referencia a la interrogante de si (como el Sr. Darwin piensa probable), los hombres y los renacuajos, aves y peces, arañas y caracoles, insectos y ostras, lirios de mar y esponjas, tuvieron un origen común en la matriz del tiempo" (Carpenter 1860b).

- "En resumen, la lucha por la existencia es un mito, y su empleo como un medio de mejoramiento es aún más mítico" (Dawson 1860).

John W. Dawson (1820-1899). Geólogo, paleontólogo y pedagogo canadiense fue Rector de la Universidad McGill, en Montreal. Sus publicaciones paleontológicas están inspiradas en sus creencias religiosas, rehusando admitir que el ser humano pueda descender por evolución de ancestros inferiores y afirmando que la especie humana apareció en la Tierra en una época muy reciente.

- "Si la notoriedad puede ser una prueba de autoría exitosa, el Sr. Darwin ha tenido su recompensa. Raramente un trabajo reconocidamente científico había atraído hacia él la atención del público tan rápidamente como El Origen de las Especies del Sr. Darwin. Su Teoría desde entonces ha llegado a ser histórica. Ella ha asumido una posición desde la cual concita la atención de todos aquellos que tienen interés en las generalizaciones de la ciencia de la naturaleza. Algunos naturalistas eminentes afirman que es incontrovertible; otros, menos intrépidos, emiten una aprobación limitada. Las Sociedades Reales la discuten en los Clubes. Es recibida con sonrisas en los salones, y desaprobada en las iglesias como 'una segunda edición de los Vestigios de la Creación [publicada en 1844 por Robert Chambers (18021883), editor y escritor que defendía la evolución, y que había causado auténtico furor en el público general. En 1860, Los Vestigios habían llegado a la $11^{a}$ edición)]. Lo menciono aquí, simplemente con el propósito de negar que [Los Vestigios] tengan algo en común que lo que caracteriza esencialmente la obra del Sr. Darwin” (Duns 1860).

- "Ningún trabajo científico publicado en este siglo ha excitado más la curiosidad general que el tratado del Sr. Darwin. Ha tenido, por un tiempo, dividido al mundo científico en dos grandes secciones contendientes: una 'darwinita' y otra 'anti-darwinita' [en inglés 'Darwinite' y 'anti-Darwinite'] son ahora las insignias de partidos científicos opuestos. Cada lado está debidamente representado. En las más altas jerarquías de la oposición contra Darwin ya han aparecido el Profesor Owen, Mr. Hopkins, Sir. B. Brodie y el Profesor Sedgwick; mientras que el Profesor Huxley, el Profesor Henslow, el Dr. Hooker y Sir Charles Lyell han dado a la nueva teoría un apoyo más o menos decidido. Nos esforzaremos muy cuidadosamente para evitar la parcialidad del partidarismo; y así nuestro objetivo no es ni atacar ni defender, sino simplemente exponer; no tendremos la necesidad de asumir el tono de mezquina hostilidad exhibida en el Edinburgh Review, o convocar desde la teología las asperezas contenidas en el Quarterly" (Fawcett 1860).

Henry Fawcett (1833-1884). Político y economista inglés. A pesar de quedar ciego en 1858 , esto no fue impedimento para convertirse en un hombre de estudio. Profesor de Economía en Cambridge (1863).

- “...El nuevo libro Sobre el Origen de las Especies por medio de la Selección Natural deja una inquietante impresión, a pesar de sus razonables y cautivantes pasajes. No nos tomó completamente de sorpresa, como a muchos de nuestros contemporáneos...Las investigaciones acerca de la sucesión de las especies en el tiempo y su actual distribución geográfica en la superficie de la Tierra conduce de todos lados y por diversos caminos hacia su origen" (Gray 1869a).

- "En su opinión no sólo los individuos de una misma especie son descendientes de un antecesor común, sino que de todas las especies emparentadas también. La afinidad de parentesco, expresión 
que todos los naturalistas usan figuradamente para expresar semejanza no derivada ni explicada entre especies, tiene un significado literal en el sistema de Darwin, que ellos apenas sospecharon, específicamente el de la herencia" (Gray 1860b).

- "Dos hipótesis sobre el origen de la diversidad existente en las plantas y animales que nos rodean dividen muy desigualmente al mundo científico. Uno asume que las actuales especies son primordiales; la otra, que son derivadas. Una, que todas las especies fueron originadas sobrenaturalmente y directamente como tales, y han continuado inalteradas en el orden de la Naturaleza; la otra, que las especies presentes aparecieron en alguna suerte de conexión genealógica con otras especies más antiguas, y que ellas llegaron a ser lo que son ahora en el transcurso del tiempo y en el orden de la Naturaleza...No estamos dispuestos ni preparados para tomar partido por o en contra de la nueva hipótesis...Los dos puntos de vista dividen muy desigualmente al mundo científico; así que los creyentes en 'el divino derecho de las mayorías' no requieren dudar qué partido tomar, por lo menos en el presente" (Gray 1860c).

Asa Gray (1810-1888). Botánico (uno de los principales del siglo XIX) y evolucionista norteamericano. Proporcionó mucha información a Darwin, especialmente en biogeografía. Apoyó las ideas de Darwin, contrariamente a su colega Louis Agassiz, pero permaneció en un devoto teísmo, tratando de reconciliar las teorías de Darwin con la teología cristiana.

- "Las interrogantes relacionadas con la naturaleza y orígenes de los fenómenos de la vida, especialmente cuando se extienden al ser humano como un ser intelectual y espiritual, naturalmente se presentan bajo dos distintos aspectos, - primero, en la directa e inmediata relación que puede conducir a la existencia y atributos de un Creador Omnisciente y Sapientísimo Gobernador del Universo, y segundo, en relación con las causas secundarias a las cuales referimos los fenómenos más comunes de la materia orgánica. El primero puede ser llamado aspecto religioso o teológico: el segundo, aspecto científico de tales interrogantes" (Hopkins 1860).

- "Nos parece que la más grande objeción que puede ser esgrimida contra la teoría [de Darwin] es su seguridad en causas naturales y posibilidad de efectuar los cambios. Estaríamos más inclinados a referir las modificaciones que las especies de animales o plantas pueden experimentar, a la voluntad directa de Dios, porque parece difícil concebir cómo un ser totalmente ignorante de su propia estructura o condiciones de vida pueda comenzar modificando su estructura, forma o hábitos para adaptarse no sólo él mismo, sino también su descendencia a nuevas formas y condiciones de vida" (Hutton 1860).

- "Cada uno de los que han leído el libro del Sr. Darwin, por lo menos ha dado una opinión sobre sus méritos y deméritos; beatos, sean laicos o eclesiásticos, lo desaprueban con una suave indiferencia que suena muy caritativa; los fanáticos lo denuncian con ignorante inventiva; damas y caballeros viejos lo consideran un libro decididamente peligroso, y aún eruditos, que no tenían más barro que arrojar, citan a escritores antiguos para demostrar que su autor no es mejor que el mismísimo simio" (Huxley 1860a).

- "No obstante, todavía no hay prueba de que, por selección, las modificaciones que tienen carácter fisiológico en las especies (i.e. cuya descendencia sea incapaz de propagación inter se) hayan sido producidas alguna vez a partir de un tronco común. El Origen de las Especies no es la primera, y no será la última de las grandes interrogantes generadas en la ciencia, lo que demandará una conciliación desde esta generación" (Huxley 1860b).

- "No obstante nuestro escepticismo sobre la teoría del Sr. Darwin, El Origen de las Especies por Medio de la Selección Natural" no es un libro que deba ser tratado con ligereza. El autor es bien conocido como un hombre de reputación en ciencia, que ha viajado sobre una gran parte del mundo, observando todo; y no ha ocultado su nombre, como algún avergonzado 'vestigiano', sino que valientemente propone su teoría, y nos dice en qué está basada" (Jardine 1869).

- "Su libro es el resultado de veinte años de paciente y constante labor, y los hechos perseverantemente recolectados se presentan de manera atractiva, y en un espíritu de inusual candor. Pero [la teoría] adopta, o al menos sugiere, opiniones sobre los modos de acción del Creador, y sobre los proce- 
dimientos de la Providencia, que son repugnantes a los más preciados sentimientos y esperanzas del hombre" (Lowell 1860).

J.A. Lowell. Estadounidense. Invitó a Jean Louis Rodolphe Agassiz a dictar un curso de Zoología en el Lowell Institute, Boston, Massachusetts. Esto motivó a Agassiz a radicarse en Estados Unidos.

- "Para usar las palabras del Sr. Darwin, "la selección natural está diaria y continuamente escudriñando en todo el mundo cada variación, aun la más leve; rechazando la desfavorable, preservando y agregando toda la que es favorable, trabajando silenciosa e insensiblemente cada vez y donde quiera que la oportunidad ofrece para el perfeccionamiento de cada ser orgánico, en relación a sus condiciones orgánicas e inorgánicas de vida'. No puedo creer en tal doctrina. He llegado a tener la opinión de que la teoría del Sr. Darwin es errónea y que puedo prescindir de cualquiera colisión entre mis inclinaciones y mis convicciones" (Murray 1860).

Andrew Murray (1828-1917). Nació en Sudáfrica, en medio de un ambiente extremadamente religioso. En 1838 se trasladó, con su padre, a Escocia. Posteriormente realizó estudios teológicos en Holanda. Dedicó su vida a predicar y a enseñar la religión. Vivió en el respeto y temor de Dios.

- "El origen de las especies es la pregunta de las preguntas en Zoología; el supremo problema que es el más constante de nuestros originales estudiosos, los más lúcidos pensadores zoológicos, y los más exitosos generalizadores nunca la han perdido de vista, al mismo tiempo que la han abordado con la debida reverencia.......El presente trabajo está ocupado por argumentos, creencias y especulaciones sobre el origen de las especies, en los cuales, como nos parece, se ha cometido el error fundamental de confundir las cosas, siendo las especies el resultado de una ley o causa secundaria" (Owen 1860). [Sir Richard Owen (1804-1892), una ambiciosa y eminente figura de la ciencia victoriana, escribió esta importante (anónima) revisión de El Origen de las Especies y de otras para la respetada Edinburgh Review; como es bien conocido, Owen vacilaba entre la aceptación o la negación de la evolución, pero estaba convencido de que los mecanismos propuestos por Darwin eran erróneos]. (Owen 1860).
Richard Owen (1804-1892). Zoólogo y paleontólogo inglés, el más prestigioso de la época victoriana. Describió y clasificó los mamíferos fósiles (cuatro volúmenes de la Zoology of the Voyage of H.M.S. Beagle, entre 1838 y 1839).

- “PPor qué cuando la voz de Dios emana de lo inanimado y dice dejemos que esto sea, no es tan fácilmente obedecida aunque esa cosa llegue a constituir un ser por desarrollo fecundo, aunque hubiera surgido de la nada o del polvo?" (Parsons 1860).

- "El libro del Sr. C. Darwin sobre la especie, que ha aparecido en Londres hace algunos meses, ha causado en Inglaterra una grande y merecida impresión. Hacía tiempo que no habíamos leído nada tan completo...El Sr. Darwin introduce un hecho importante que es la lucha por la existencia...Y la elección (sic) natural (selección natural) que juega para el perfeccionamiento de las razas salvajes el mismo rol que la elección del criador de ganado para las razas domésticas" (Pictet 1860).

Francois Jules Pictet (1809-1872). Zoólogo y paleontólogo suizo. Entomólogo eminente del siglo XIX. Junto con Alfred Edwin Earon es uno de los primeros especialistas en efemerópteros. En paleontología es autor de Traité élémentaire de Paléontologie, ou Histoire naturelle des animaux fossiles considerés dans leur rapports zoologiques et géologiques (1846).

- "Su presente volumen sobre "El Origen de las Especies" es el resultado de muchos años de observación, reflexión y especulación; y claramente es considerada por él como el 'opus' sobre el que descansará su futura fama...; es verdad que él lo presenta modestamente como el mero precursor de un volumen mayor..., destinado a suministrar los hechos que apoyen el argumento completo del presente ensayo. [La materia de que trata] es del más profundo interés...para todo el que esté interesado en la historia del hombre y las relaciones de la naturaleza alrededor de él y a la historia y plan de la creación. [Si fallan algunas de las proposiciones que establece el autor] la teoría completa del Sr. Darwin se cae en pedazos. .. Asumiendo que el hombre como selector [en los animales domésticos] puede hacer mucho en un tiempo limitado, el Sr. Darwin argumenta que la Naturaleza, un poder más potente, 
actuando sobre extensos períodos de tiempo, puede hacer más...No hay límites a su exuberante fantasía [como cuando] indica la aplicación de su sistema a los animales inferiores y hasta el hombre mismo... Tales suposiciones son en su mayoría oprobiosas e injuriosas para la ciencia...El Sr. Darwin escribe como un cristiano, y no dudo que lo sea...No simpatizamos con aquellos que exponen algunos hechos o supuestos hechos de la naturaleza, ... porque creen que contradicen lo que les parece a ellos que es enseñado por la Revelación...El que está tan seguro como él de su propia existencia, que el Dios de la Verdad es también el Dios de la Naturaleza y el Dios de la Revelación, no puede creer como posible que Su voz...pueda discrepar, o engañar a sus criaturas (Wilberforce 1860).

Samuel (Bishop) Wilberforce (1805-1873). Obispo de Oxford, contemporáneo de Darwin.

Principal representante antidarwinista del clero de Inglaterra. Aunque no entendía nada de temas científicos, excepto de matemáticas, de las cuales había hecho estudios, su participación en un debate con Thomas Henry Huxley en la reunión en Oxford en 1860, de la British Association for the Advancement of Science ha pasado a la historia con el carácter de leyenda: el clérigo no científico fue derrotado, ante un auditorio de 700 personas, por el científico, en lo que fue calificado como el gran enfrentamiento entre la religión y la ciencia.

- "El Sr. Darwin...cree...que todas las especies (incluido el hombre), pueden haber derivado, cada uno a su turno, de aquellas bajo ellas por el mero 'poder seleccionador de la naturaleza', que se supone ha trabajado continuamente a través de incontables años, rechazando (por inevitable aniquilamiento) a los individuos más débilmente desarrollados y enfermos que en todas partes han existido, y preservando cada pequeña modificación que se presentó de tiempo en tiempo (en la gran "lucha por la vida" que siempre ha ocurrido entre los seres orgánicos, para acudir en beneficio de su poseedor, y transmitido, por la ley de la herencia, a la siguiente generación, incrementándose en la dirección sana hasta que al fin, en el curso de los siglos, las diversas razas han llegado a modificarse tanto en estructura (y que, además, intermitentemente, o como fuera, en camino, de acuerdo a su posición, o avance, en el pedigrí animal), como para haber asumido las diversas formas, pasadas y presentes, que los naturalistas han descrito con el nombre de 'especies'. Los fósiles de cada formación geológica, en su opinión, 'no marcan un nuevo y completo acto de creación, sino sólo una escena ocasional, tomada casi al azar, en un drama de cambio lento" (Wollaston 1860).

Thomas Vernon Wollaston (1822-1878). Entomólogo inglés, autor de Coleoptera SanctaeHelenae. London. 256 pp., que trata recolecciones de Darwin.

Y siguieron apareciendo artículos y libros dedicados a la revisión de las ideas de Darwin; y el autor siguió respondiendo a las objeciones, tratando de convencer a los opositores.

La vida posterior de Darwin fue prácticamente dedicada a la defensa de su libro en las sucesivas ediciones, revisadas, corregidas, modificadas, que lo hacen expresar "este volumen completo es un largo argumento" (Capítulo XV. Recapitulación y conclusión).

\section{CONCLUSIONES}

- Dificilísimo hubiera sido lograr consenso en un enfrentamiento entre el conocimiento religioso y el conocimiento científico, entre la interpretación literal de la Biblia y los hechos que iban revelando, materiales e interpretación, los adelantos de la ciencia, con excepciones, por supuesto. (El Origen de las Especies "está ocupado por argumentos, creencias y especulaciones" [Owen 1860]; "sugiere opiniones... que son repugnantes a los más preciados sentimientos y esperanzas del hombre" [Lowell 1860]).

Y más aún después de la publicación de El Origen del Hombre (1871). Ya en Origen de las Especies Darwin había adelantado que "en el futuro... se proyectará mucha luz sobre el origen del hombre y sobre su historia" (Capítulo XV).

Darwin cree "que todas las especies (incluido el hombre), pueden haber derivado de aquellos bajo ellos...(Wollaston 1860).

No podía aceptarse un nexo entre el ser humano y el simio. Como recuerda Huxley (1860a), incluso algunos eruditos de la época llegaron a afirmar que el autor no era mejor que el mismísimo simio; en el célebre debate en Oxford (1860), el obispo Wilberforce, para ridiculizar las ideas evolucionistas 
de Huxley le preguntó a éste si descendía de un simio por parte de abuelo o de abuela; se corrió la noticia, inexacta, de que Huxley había respondido que preferiría ser simio antes que obispo (Milner 1995).

-Estaban enfrentados dos aspectos fundamentales del ser humano: el religioso o teológico y el científico (Hopkins (1860), aunque Darwin no viera " ninguna razón válida para que las opiniones expresadas en este libro ofendan los sentimientos religiosos de nadie" (Capítulo XV).

Darwin elogia al zoólogo inglés George J. Mivart (1827-1900), fervoroso católico, enconado enemigo de las ideas de Darwin y finalmente declarado darwinista (Capítulo VII. Objeciones diversas a la teoría de la selección natural), que aparentemente estaba logrando puntos de acuerdo entre religión y ciencia. La única objeción de Mivart, profesor de la Universidad Católica de St. Mary, era la de que había que agregar la intervención de Dios para infundirle un alma al cuerpo humano evolucionado a partir de un antepasado simioide. En 1900 fue excomulgado.

- El 21 de abril de 1869 se fundó en Londres la Metaphysical Society, con los objetivos de examinar y debatir los diferentes puntos de vista sobre la fe, las creencias, la ciencia, Dios, la moralidad, los milagros, la verdad y las bases de todo tipo de conocimiento. Se reunieron una vez al mes, durante doce años, disolviéndose por no haber logrado ningún objetivo general y perdurable (Milner 1995).

- En realidad, parece que cuando Charles Darwin publicó El Origen de las Especies, creó "un poder activo o divinidad", como lo expresa, brevemente (Capítulo IV).

Así, tal como ha ocurrido en la historia de la humanidad, grupos humanos (en este caso Darwin y Wallace) crean una divinidad (en este caso la selección natural) cuyas directrices emanan de un libro sagrado (en este caso El Origen de las Especies), que es la base de cada religión (en este caso el darwinismo). Y para completar la analogía, siempre la divinidad es mencionada recurrentemente. En este caso la selección natural es nombrada 50 veces.

Y, por supuesto, siempre han existido pugnas entre las religiones, aunque el darwinismo nunca, obviamente, hubiera pretendido ser una religión.
- A pesar del tiempo transcurrido, todavía las ideas de Darwin siguen produciendo ruido. En este año, 2009, "el año de Darwin", en el bicentenario de su nacimiento (1809) y el sesquicentenario de la publicación de El Origen de las Especies (1859) han aparecido en los periódicos ("Cartas al Director") opiniones discordantes con Darwin, no con la intensidad de antes, pero que reflejan la perduración de antiguas ideas.

Charles Darwin, que publicó 17 libros en 21 volúmenes (con un total de 9.000 páginas impresas), más otras 1.000 páginas en artículos científicos, prácticamente pasó a la posteridad por el recuerdo de las polémicas y controversias, mayoritariamente surgidas en torno a su Origen de las Especies, obra que defendió y enmendó a lo largo de toda su vida, hasta que ya no tuvo nada más que decir.

El ser humano posee una religiosidad natural. La causa principal de la reacción prototípicamente religiosa es la inmensidad del universo, ya que aunque el hombre pueda aprender mucho acerca del universo, nunca logrará abarcarlo todo, por lo cual se promoverá en él una respuesta denominada "el sentido del misterio". El espíritu del hombre permanecerá inalterable aunque las religiones cambien en un proceso de adaptación. Esta es la razón de la religión (Ferm 1964).

\section{LITERATURA CITADA}

Agassiz, J.L.R. 1860. [Review of] On the Origin of Species. American Journal of Sciences and Arts (series 2) 30 (July):142-154. Online: http://darwin-online.org.uk/content/ frameset? itemID $=\mathrm{A} 45 \&$ viewtype $=$ image $\&$ pageseq $=1$ 24/04/2007.

Bowen, F. 1860. [Review of] On the Origin of Species by Means of Natural Selection. North American Review 90:474-506. Online: http://darwin-online. org.uk/content/ fram eset?itemID $=\mathrm{A} 15 \&$ viewtype $=$ text\&pages eq=1 04//05/2007.

Bronn, H.G. 1860. [Review of] Ch. Darwin: On the Origin of Species by means of Natural Selection, or the preservation of favoured races in the struggle for life (502 pp. $8^{\circ}$, London 1859). Neues Jahrbuch für Mineralogie, 
Geognosie, Geologie und Petrefaktenkunde. 112-116. 04/05/2007.

Carpenter, W.B. 1860a. The theory of Development in Nature. British and Foreign Medico- Chirurgical Review 25(April):367-404. Online: http://darwin- online.org.uk/content/frame set?itemID=A46\&viewtype=image\&pages eq=1 25/04/2007.

Carpenter, W.B. 1860b. Darwin on the Origin of the Species. National Review 10:188214. Online: http://darwin-online.org.uk/ content/frameset?itemID=A17 \&viewtype =text\&pageseq=1 25/04/2007.

Darwin Online 2009. Online: http://darwin-online. org.uk. 20/09/2009.

Dawson, J.W. 1860. [Review of] Darwin on the origin of species by means of natural selection. Canadian Naturalist and Geologist 5:100-120. Online: http://darwin-online.org.uk/content/ frameset?itemID=A47\&viewtype=image\&pa geseq $=1$ 04/05/2007.

[Duns, J.] 1860.. [Review of] On the Origin of Species. North British Review 32 (1860 May):455-486. Online: http://darwin-online. org.uk/content/frameset?itemID=A48viewtype =image \&pageseq=1 25/04/2007.

Fawcett, H. 1860. A Popular Exposition of $\mathrm{Mr}$ Darwin on the Origin of Species. Macmillan's Magazine (3 December):81-92. http://darwinonline.org.uk/ content/ frameset? itemID $=\mathrm{A}$ 23\&viewtype=text\&pageseq=1 04/05/2007.

Ferm, V. 1964. Religión. In: Gran Enciclopedia del Mundo 16: 373-377. Durvan, S.A. de Ediciones - Bilbao.

Ghiselin, M.T. \& P. Goldie 1997. A Guide to Darwin Studies. Introduction and study guide. Printed from The Darwin CD-ROM, $2^{\text {nd }}$ Edition.

Ghiselin, M.T. \& B. Pope 1997. The Darwin biogeographical dictionary. Printed from The Darwin CD-ROM, $2^{\text {nd }}$. Edition.

Gray, A. 1860a. [Review of] On the Origin of Species. Atlantic Monthly 6 (1860 July):109-116, 229-239. Online: http:// darwin- online.org.uk/content/frameset? itemID=A49 \&viewtype $=$ image\&pageseq $=1$ 26/04/2007.

Gray, A. 1860b. Review of Darwin's theory on the origin of species by means of natural selection. American Journal of Science and
Arts (series 2) 29 (March):153-184. On line: http://darwin-online.org.uk/content/frameset? itemID $=\mathrm{A} 50$ \&viewtype $=$ image $\&$ pageseq $=1$ 26/04/2007.

Gray, A. 1860c. Darwin and his reviewers. Atlantic Monthly 6 (1860 October):406-425. On line: http://darwin-online.org.uk/content/frameset ?itemID $=\mathrm{A} 51$ \&viewtype $=$ image $\&$ pageseq $=1$ 27/04/2007.

Higgins, H.H. 1860. On Darwin's theory of the "Origins of Species". Proceedings of the Literary and Philosophical Society of Liverpool 15:42-49. Online: http://darwin-online.org. $\mathrm{uk} /$ content/frameset?itemID=A52\&viewtyp $e=$ image\&pageseq $=1$ 26/04/2007.

Hopkins, W. 1860. Physical Theories of the Phenomena of Life. Fraser's Magazine 61(June):739-752; 62 (July):74-90. Online: http:// darwin- online.org.uk/content/ frameset?it emID $=$ A53\&viewtype $=$ image $\&$ pagese $=1$ 24/04/2007.

Hutton, F.W. 1860. [Review of] On the Origin of Species. The Geologist 3(December) :464-472. Online: http://darwin-online.org.uk/content/ frameset?itemID $=$ A55 \& viewtype=image \&pageseq=1 04/05/2007.

Huxley, T.H. 1859a. Darwin on the origin of species The Times (26 December):8-9. Online: http:darwin-online.org.uk/content/frameset ?itemID=168\&viewtype=text\& pageseq $=1$.

Huxley, T.H. 1859b. Time and Life: Mr. Darwin's "Origin o Species". Macmillans Magazine 1:142-148. Online: http://darwin- online.org. $\mathrm{uk} /$ content/frameset? itemID $=\mathrm{A} 438$ viewtyp $e=$ image\&pageseq $=1$ 24/04/2007.

Huxley, T.H. 1860a. Darwin on the origin of Species. Westminster Review 17 (n.s.):541570. Online: http://darwin-online.org. $\mathrm{uk} /$ content/frameset? item $\mathrm{ID}=\mathrm{A} 3 \&$ viewtype=text\&pageseq=1 25/04/2007.

Huxley, T.H. 1860b. On species, races and their origin. Journal Proceedings of the Royal Institution of Great Britain 3(February):195-200. Online: http://darwin-online.org.uk/content/ frameset?itemID=A56\&viewtype=image\&p ageseq=1 26/04/2007.

Jardine, W. 1860. [Review of] On the origin of species. Edinburgh New Philosophical Journals n.s. 11:280-289. Online: http://darwin-online. 
org.uk/content/frameset? itemID $=\mathrm{A} 57 \&$ view type=image\&pageseq $=1$ 26/04/2007.

Josa i Llorca. 1008. Introducción. In Darwin, C. El Origen de las Especies, pp. 13-57. J.

Josa i Llorca (Ed.). Edición conmemorativa. El Origen de las Especies. Editorial Espasa-Calpe S.A. 695 pp.

Lowell, J.A. 1860. [Review of] Darwin's Origin of Species. Christian Examiner and Theological Review 6:449-464. Online: http://darwin- online.org.uk/content / fra meset?itemID=A22\&viewtype\&pageseq $=1$ 04/05/2007.

Milner, R. 1995. Diccionario de la Evolución. Bibliograf, S.A. España. 685 pp.

Murray, A. 1860. On Mr. Darwin's theory of the origin of species. Proceedings of the Royal Society of Edinburgh 4:274-291. Online: http://darwin-online.org.uk/content/frames et?itemID=A14\&viewtype=text\&pageseq $=1$ 24/04/2007.

Owen, R. 1860. [Review of Origin \& other works]. Edinburgh Review 111:487-532. On line: http://darwin-online.org.uk/content/frameset? itemID $=\mathrm{A} 30$ \&viewtype $=$ image\&pageseq $=1$ 24/04/2007.

Parsons, T. 1860. [Review of] On the Origin of Species. American Journal of Science and
Arts (series 2) 30(July):1-13. On line: http:// darwin- online.org.uk/content /frameset?it emID=A60\&viewtype=image\&pageseq $=1$ 04/05=2007.

Pictet, F.J. 1860. Sur l'origine de l'espèce par Charles Darwin. Archives des Sciences, Bibliothèque Universelle (Ser.2) 7 (March):233-255. Online: http://darwin-online.org.uk/content/framese t?itemD $=\mathrm{A} 61 \&$ viewtype $=$ image \&pageseq $=1$ $07 / 05=2007$.

Smith, K.G.V. /Ed.) 1987. Darwin's Insects. Bulletin of the British Museum (Natural History, Historical Series 14(1):1-143.

Wilberforce, S. 1860. [review of] On the Origin of Species, by means of Natural Selection; or the Preservation of Favoured Races in the Struggle for Life. Quarterly Review 108:225-264. Online: http://darwin-online. org.uk/content/frameset?itemID=A19 \& viewwtype=text\&pageseq=1 04/05/2007.

Wollaston, T.V. 1860. [Review of] On the Origin of Species by means of Natural Selection; or, the Preservation of Favoured Races in the Struggle for Life. Annals and Magazine of Natural History 5:132-143. Online: http:// darwin-online.org. uk/ content/frameset? item $\mathrm{ID}=\mathrm{A} 18 \&$ viewtype $=$ text $\&$ pageseq $=1$ 04/05/2007. 